\title{
Photon Structure and Behavior
}

\author{
Jianping Mao
}

\section{ABSTRACT}

One of possibilities for that why like charges repel and opposite charges attract was a photon possessing negative and positive two poles that with an up or down spin frequency - any integer - offers a fresh insight into photon energies.

Keywords: charge repel-attract, force lines, spin frequency, photon structure.

Published Online: October 31, 2021

ISSN: $2684-4451$

DOI : 10.24018/ejphysics.2021.3.5.110

\section{J. Mao*}

(e-mail: mjp00951@163.com)

*Corresponding Author

\section{INTRODUCTION}

Light was wave [1] or particle [2]? Now the argument is explained to display wave-particle duality in uncertainty principle of modern physical theories. While in here prefers particle to wave that light not only is made of particles photons [3]-[7], but also, they could own two opposing charge poles that gave rise to charge r-a (repel-attract) property; what is more, it is accompanying a spin can provide an easier sketch on an origin of electromagnetic frequency (Fig. 1).

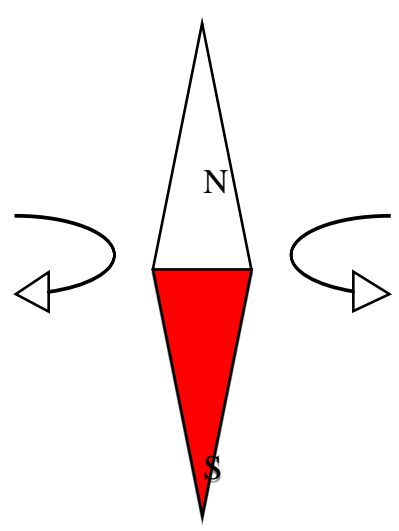

Fig. 1. Photon image and spin.

A neutral photon (boson) seems like a compass or a fermionic dyon having both positive (+, N: north pole or norton - north particle) and negative (-, $\mathrm{S}$ : south pole or souton - south particle) charges that around its NS axis could emerge two spin (not only \pm 1 , but also any integer as frequency) directions.

\section{SUPPOSITIONS AND DisCUSSIONS}

\section{A. Photon Structure}

This photon image came from a problem that like charges repel each other and opposite charges mutually attract, which was often in my mind for a few decades. Why it is so that has not a deeper explanation on it up to now, to the best of my knowledge. Here a consideration is that either electric or magnetic field is made up of lines of force [8] and the lines consist of photons; if these photons own an intrinsic configuration that occurs a N-S pair analogous to an electronpositron one but smaller, a fermionic dyon or skyrmion [9][12], and unable to be broken almost in any condition, which likely was anisotropic, oscillating and deformable (Fig. 1), and then line them (looking like strings) [13]-[16] able to create charge r-a phenomena (Fig. 2a-b).

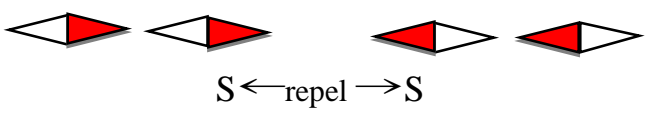

(a)

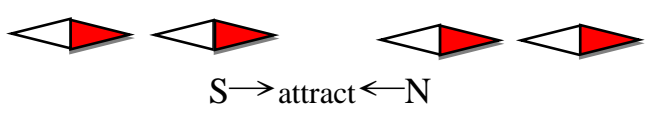

(b)

Fig. 2. Force line r-a occurrence of two magnets. (a) Nose to tail photons corresponds to Faraday's illuminating a magnetic curve or line of force, no matter in magnetic or electric fields, subsequently displays like (negative) charges repulsion.

(b) Opposing (negative and positive) charges attraction.

However, it was surprised that when I skimmed a paper " $A$ Photon is a Magnetic Dipole" and its references [17], [18] on viXra in Aug. 3, 2021, which is nearly comparable to my and stimulated me ahead of time to write this paper. For he used electromagnetic r-a phenomena to elucidate double-slit experiment I agree partly, since lattice materials of a doubleslit, and others we unclear, might be involved.

\section{B. Photon Spin and Energy}

A photon energy is generally related to atomic or nuclear energy levels when a transition is from excited to ground state that are potential energies and their pictures a bit was unphysical (see also an elegant tangible nuclear model of 1- 
118 elements and their different isotopes [19]), which the energy span is from radio waves to gamma rays $\left(\sim 10^{-9}-\right.$ $10^{6} \mathrm{eV}$ or $\sim 10^{6}-10^{20} \mathrm{Nz}$ ). A problem is that when a photon has been emitted afterward it became a free photon that how to express its kinetic energy?

To express real meaning of a photon energy, a feasible way is to assume that its frequency could roughly be regarded as another expression of its spin (somewhat like a spinor [20][22]) that was variable (any integer, approximately equal to its angular momentum plus its orbital angular momentum in modern physical concept), and exhibited a certain direction known as left-handed and right-handed - a spin photon probably linking to a spin of our universe and as a consequence leads to an asymmetric world that matter is more much than antimatter [23], or up and down that a photon has two possible polarization states [24].

That is:

$$
E=h c / \lambda=h v=h f
$$

giving

$$
f=s f
$$

So:

$$
E=h s_{f}
$$

where are: $E$, photon energy; $h$, Planck constant; $c$, light speed; $\lambda$, wavelength; $f / v$, frequency; and $s$, spin frequency. Obviously, it is accessible to understand photon energies (Fig. 1).

\section{Photon Mass and Charge}

This scheme is based on light wave theory that still used wavelength or frequency term. A photon energy, on the other side, perhaps can't completely exclude that might partly result from its mass; however, our world displays a certain shape and size that is made of material. Commonly, an ordinary object kinetic energy is:

$$
E=m v^{2} / 2,
$$

which its energy is proportional to its mass. But in a photon energy:

$$
E=m c^{2}
$$

photon speed $c$ is constant in vacuum and mass $m$ is massless.

Accordingly, trying to seek a practicable means is that a photon may occur an intrinsic (rest) mass $m$, but could increase to $n m, n=1,2,3 \ldots$, like Bose-Einstein condensation [25] and a big (multi) photon, even so large as a neutron, a mini black hole [26], [27] (a neutrino might be a mini black hole?), or a black hole (needless to say its photon spherecircle [28]), that its properties are same as a single photon. This is due to that electron and positron pairs can be annihilated into two photons

$$
\mathrm{e}^{-}+\mathrm{e}^{+} \rightarrow \gamma+\gamma
$$

in free space, or contrary

$$
\gamma \rightarrow \mathrm{e}^{-}+\mathrm{e}^{+}
$$

adjacent atomic nuclei [29], [30], implying that electronpositron pair came mostly from one of nucleons of the atomic nuclei, and a photon existing mass and charge, no matter more or less, is necessary.

Hence, a photon could have mass and charge, and, on the other side, its particle characteristic is more prior than of wave that merely is a show of its mass-charge field.

For a long time, an attempt is using photons, which not only is force carrier but also a mere basic particle was assumed that all of others were composite [23], to simulate structures of electron and positron after that to neutron and proton [31], i.e.:

$$
\gamma \rightarrow \mathrm{e}^{-}+\mathrm{e}^{+} \rightarrow \mathrm{n} \rightarrow \mathrm{p}^{+}+\mathrm{e}^{-}
$$

Nevertheless, a clear map has not been drawn so far, where an embryonic idea in Fig. 3a-c shows some possible subparts of these particles, along with they grow to various size shapes that can match partons, or quarks and leptons to some degree.

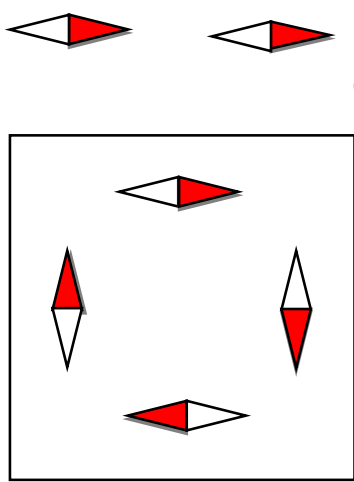

(b) (a)
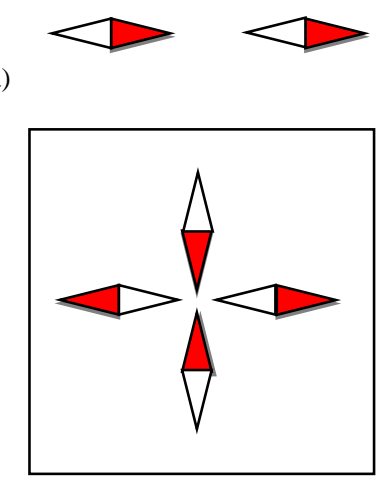

(c)
Fig. 3. Photon forming open, closed and quadrupole "strings". Photons could be configured to produce various pieces of stable and unstable fundamental particles. (a) 1-D (dimensional), linear. (b) 2-D, circular. (c) 3-D (Quadrupole photons / magnets), tetrahedral.

In addition, about mass and charge a topic is their relationship that mass has $F_{g}$ (gravitational-) and charges have $F_{r}$ (repelling-) and $F_{a}$ (attracting-force), noticing that among four fundamental (gravitational, electromagnetic, weak, and strong) forces solely electromagnetic one can occur repelling characteristic, including of proton-proton.

Here a guess is that if there is a balance (cross) point, $10^{-15} \mathrm{~m}$ (i.e., nucleon scale, Fig. 4):

$$
F_{r}=F_{a},
$$

when a distance is larger than this value:

$$
F_{r}<F_{a}
$$

otherwise, reverse. Thus:

$$
F_{g}=F_{a}\left(\mathrm{e}^{-} \rightarrow \longleftarrow \mathrm{e}^{+}\right)-F_{r}\left(\mathrm{e}^{-/+} \longleftrightarrow \mathrm{e}^{-/+}\right),
$$

it is meaning that gravitational and electromagnetic forces 
may be identical, which the both might slightly deviate from inverse-square law.

In the light of this, so far as to say other three forces were in different scale show of electromagnetic one (Fig. 4). However, it is uncertain that whether some physical laws and constants are invariant, or not [32]-[35].

Finally, a difficult problem in next step is that how to estimate a value of a photon mass and charge, and then to construct electron or positron, but in a what case uncharged photons (bosons, a fermionic dyon) can form charged particles (fermions, that vacuum polarization can? [36], [37]), very key, and how many photons ( $\mathrm{N}$ or $\mathrm{S}$ ) need to build an elementary charge ( $e$, electron or positron, its radius: $\sim 10^{-22} \mathrm{~m}$ [38]), if charge is conservation (Fig. 5a-b; incidentally, this resetting figure before had been removed, but when see a resemblance - Fig. 3a in [39]), which their present rough values are: $\mathrm{m}_{\gamma} \lesssim 10^{-14} \mathrm{eV} / \mathrm{c}^{2}, \mathrm{q}_{\gamma}<1 \times 10^{-35} e$, respectively [40]-[42]. A photon charge is about $e / 10^{35}$ that is net and according to a quark charge was $e / 3$ or $2 e / 3$, absolute value, a $\mathrm{N}$ or $\mathrm{S}$ in a photon was $e / 4, e / 8$, etc.?

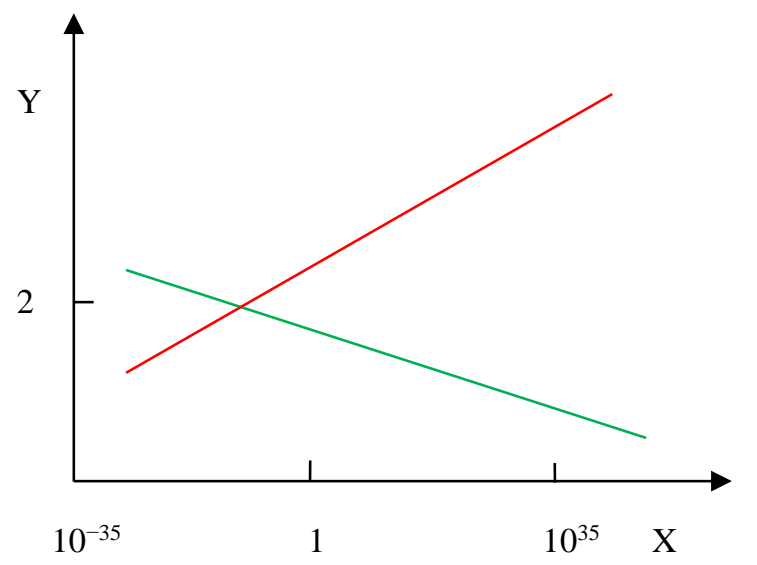

Fig. 4. Exponent as a function of distances.

This only is conceptual, not precise values. Red and green lines represent repelling and attracting forces, respectively; and distance $m$ is at $\mathrm{X}$ axis and exponent $n$ in $F=G m_{1} m_{2} / r^{n}$ and $F=k q_{1} q_{2} / r^{n}$ is at $\mathrm{Y}$ axis that both gravitational and Coulomb constants: $G\left(6.674 \times 10^{-11} \mathrm{~m}^{3} \mathrm{~kg}^{-1} \mathrm{~s}^{-2}\right)$ and $k$ $\left(\left(8.988 \times 10^{9} \mathrm{~N} \mathrm{~m}^{2} \mathrm{C}^{-2}\right)\right.$ would be integrated into a stronger (fine structure constant, $\alpha \sim 1 / 137$, as a reference?) "nuclear" one.

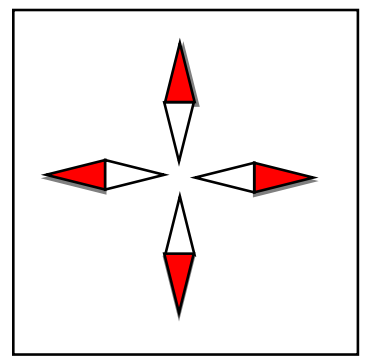

(a)

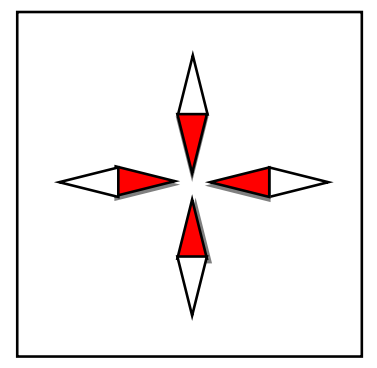

(b)
Fig. 5. Structure of negative and positive elementary charges. In a what condition photon gravity can hold these repelling photons and how many it need in (a) a negative- and (b) a positive-charges, unless in a scale, $\sim 10^{-20} \mathrm{~m}$, like charges attract somewhat similar to Cooper pairs [45] and opposing charges repel?

However, perhaps there was a certain mass-to-charge ratio (m/Q) [43] that, e.g., 4 photon masses equal to 4 norton plus souton charges:

$$
4 \mathrm{~m}_{\gamma}=4\left(\mathrm{Q}_{\mathrm{N}}+\mathrm{Q}_{\mathrm{S}}\right)
$$

although net charges of 4 photons are zero that is parallel to a neutron containing proton and electron, implying that a photon could sever as two tiniest units of mass and charge, or mass could stand for charge, vice versa that is no difference between them to some extent, if possible.

What tending to think a picture of that cosmos was shaped solely by photons (bosons, matter) and electromagnetic force (interaction) is too simple to likely?

Now discussion has reached photon level, a smallest overt stable particle that unlikely was bigger than an electron we known, that might be an upper limit of that we can do. Namely to find a latent sub-photon particle ( $\mathrm{N}$ and $\mathrm{S}$, or force particles, e.g., graviton that its mass: $m_{\mathrm{g}} \leq 7.7 \times 10^{-23} \mathrm{eV} / \mathrm{c}^{2}$ [44]; relative speaking, gluons, $\mathrm{W}$ and $\mathrm{Z}$ bosons were too large) getting more information seems impossible, despite need for a deeper reason upon charge $r$-a character, and others. Will string $\left(\sim 10^{-35} \mathrm{~m}\right.$, Planck length) be?

\section{CONCLUSION}

Electromagnetic r-a phenomenon existence seems to indicate that a photon not only was a point-like particle, but also had two opposing charge endpoints that can occur up or down spin with a variable frequency, which gave rise to photon energies of large span range $\left(\sim 1-10^{15}\right)$.

\section{ACKNOWLEDGMENT}

The author would like to thank Mr. Benlin Liu for helpful discussions.

\section{REFERENCES}

[1] R. Hooke, Micrographia: or some physiological descriptions of minute bodies made by magnifying glasses with observations and inquiries thereupon... London: Royal Society of London. 1667. ISBN 978-0486-49564-4.

[2] I. Newton, [1730]. Opticks (4 ${ }^{\text {th }}$ ed). Dover, NY: Dover Publications. Book II, Part III, Propositions XII-XX, Queries 25-29. 1952. ISBN 978-0-486-60205-9.

[3] M. Planck, "Ueber das Gesetz der Energieverteilung im Normalspectrum (On the Law of Distribution of Energy in the Normal Spectrum)," Annalen der Physik, vol.4 (3), p. 553, 1901.

[4] A. Einstein, "Über einen die Erzeugungund Verwandlung des Lichtes betreffenden heuristischen Gesichtspunkt, "Annalen der Physik, vol. 17 (6), pp. 132-148, 1905.

[5] R. Millikan, "A Direct Determination of "h"," Phys. Rev., vol.4 (1), pp. 73-75, 1914.

[6] G. N. Lewis, "The conservation of photons," Nature, vol. 118, pp. 874$875,1926$.

[7] A. H. Compton, "A Quantum Theory of the Scattering of X-Rays by Light Elements." Phys. Rev., vol.21 (5), pp. 483-502, 1923.

[8] D. Peter, The Philosopher's Tree: A Selection of Michael Faraday's Writings. CRC Press. ISBN 0-7503-0570-3. p. 125, 1999.

[9] J. Schwinger, “A Magnetic Model of Matter,” Science, vol. 165 (3895), pp. 757-761, 1969.

[10] E. Witten, "Dyons of Charge e $\theta / 2 \pi$," Phys. Lett. B., vol. 86 (3-4), pp. 283-287, 1979.

[11] T. Skyrme, "A unified field theory of mesons and baryons," Nuclear Physics, vol.31,pp. 556-569, 1962.

[12] A. Usama, S. Henk, "Skyrmions in a ferromagnetic Bose-Einstein condensate," Nature, vol.411 (6840), pp. 918-20, 2001.

[13] M. Zeeya, "Collaborative physics: string theory finds a bench mate," Nature, vol.478 (7369), pp. 302-304, 2011.

[14] R. Lisa, S. Raman, "An alternative to compactification," Phys. Rev. Lett., vol.83 (23), pp. 4690-4693, 1999.

[15] S. Ashoke, "Strong-weak coupling duality in four-dimensional string theory," International Journal of Modern Physics A., vol. 9 (21), pp. 3707-3750, 1994. 
[16] H. Chris, T. Paul, "Unity of superstring dualities," Nuclear Physics B., vol. 4381 (1), pp. 109-137, 1995.

[17] K. L. Rajpal, A Photon is a Magnetic Dipole, viXra 2105.0017, 2021.

[18] M. W. Evans, "The Elementary Static Magnetic Field of the Photon," Physica B, vol. 182, pp. 227-236, 1992.

[19] J. Mao, "The Periodic Table Possible Coincided with an Unfolded Shape of Atomic Nuclei, "Applied Physics Research, vol. 9 (6), p. 47, 2017.

[20] A. Oknin'ski, On spin-charge separation, viXra 2107.0178, 2021.

[21] P Czajka, T. Gao, M. Hirschberger, P. Lampen-Kelley, A. Banerjee, J. Yan, D. G. Mandrus, S. E. Nagler, N. P. Ong, "Oscillations of the thermal conductivity in the spin-liquid state of $\alpha-\mathrm{RuCl}_{3}$, "Nature Physics, vol.17, pp. 915-919, 2021.

[22] Y. Jompol, C. J. B. Ford, J. P. Griffiths, I. Farrer, G. A. C. Jones, D. Anderson, D. A. Ritchie, T. W. Silk, A. J. Schofield, "Probing SpinCharge Separation in a Tomonaga-Luttinger Liqui," Science, vol. 325 (5940), pp. 597-601, 2009.

[23] J. Mao, "Cosmic Background," European Journal of Applied Physics, vol. 3(1), pp. 67-70, 2021.

[24] M. D. Schwartz, Quantum Field Theory and the Standard Model. Cambridge University Press. p. 66. ISBN 978-1-107-03473-0, 2014.

[25] M. H. Anderson, J. R. Ensher, M. R. Matthews, C. E. Wieman, E. A. Cornell, "Observation of Bose-Einstein Condensation in a Dilute Atomic Vapor," Science, vol. 269 (5221), pp. 198-201, 1995.

[26] S. W. Hawkin, "Gravitationally collapsed objects of very low mass," Monthly Notices of the Royal Astronomical Society, vol. 152, pp. 75, 1971.

[27] H. J. Treder, "The planckions as largest elementary particles and as smallest test bodies," Foundations of Physics. Springer, vol.15 (2), pp. $161-166,1985$.

[28] R. C. Claes, "Using the Uncharged Kerr Black Hole as a Gravitational Mirror," General Relativity and Gravitation, vol.29 (4), pp. 445-454, 1997.

[29] J. Eichler, "Electron-positron pair production in relativistic ion-atom collisions," Phys. Lett. A., vol. 347 (1-3), pp. 67-72, 2005.

[30] J. H. Hubbell, "Electron positron pair production by photons: A historical overview," Radiation Physics and Chemistry, vol. 75 (6), pp. 614-623, 2006.

[31] G. A. Miller, "Charge Densities of the Neutron and Proton," Phys. Rev. Lett., vol. 99 (11), pp. 112001, 2007.

[32] P. A. M. Dirac, "A New Basis for Cosmology," Proceedings of the Royal Society A., vol. 165 (921), pp.199-208, 1938.

[33] T. Teller, "On the change of physical constants," Phys. Rev., vol.73 (7), pp. 801-802, 1948.

[34] D. Michael, "Trialogue on the number of fundamental constants," Journal of High Energy Physics, 2002 (3): 023.

[35] J. P. Uzan, "The fundamental constants and their variation, Observational status and theoretical motivations," Reviews of Modern Physics, vol.75 (2), pp. 403, 2003.

[36] P. A. M. Dirac, "Discussion of the infinite distribution of electrons in the theory of the positron," Cambridge Phil. Soc., vol. 30 (2), pp. 150163,1934 .

[37] I. Levine et al., "Measurement of the Electromagnetic Coupling at Large Momentum Transfer," Phys. Rev. Lett., vol.78 (3), pp. 424-427, 1997.

[38] H. Dehmelt, "A Single Atomic Particle Forever Floating at Rest in Free Space: New Value for Electron Radius," Physica Scripta,T22, pp. 102 $110,1988$.

[39] V. Vaguine, Toward Viable Electron Model based on Classical Electrodynamics, viXra 2109.0135,2021.

[40] C. Amsler et al. "Review of Particle Physics: Gauge and Higgs bosons," Phys. Lett. B., vol.667 (1), pp. 1, 2008.

[41] V. V. Kobychev, S. B. Popov, "Constraints on the photon charge from observations of extragalactic sources," Astronomy Letters, vol.31 (3), pp. 147-151, 2005.

[42] E. Williams, J. Faller, H. Hill, "New Experimental Test of Coulomb's Law: A Laboratory Upper Limit on the Photon Rest Mass, " Phys. Rev. Lett., vol.26 (12), pp. 721, 1971.

[43] J. J. Thomson, "Cathode Rays," Philosophical Magazine, vol. 44, p. 293, 1897.

[44] B. P. Abbott et al., "GW170104: Observation of a 50-Solar-Mass Binary Black Hole Coalescence at Redshift 0.2," Phys. Rev. Lett., vol. 118 (22), p. 221101, 2017.

[45] L. N. Cooper, "Bound electron pairs in a degenerate Fermi gas," Phys. Rev., vol.104 (4), pp. 1189-1190, 1956. 